\title{
Dependence of Nanoelectronic-Structure Defect Detection by Cathodoluminescence on Electron Beam Current
}

\author{
M. Pluska, A. Czerwinski, J. Ratajczak, A. Szerling and J. Kątcki \\ Institute of Electron Technology, al. Lotników 32/46, 02-668 Warsaw, Poland
}

\begin{abstract}
The dependence of defect detection by cathodoluminescence in a scanning electron microscope on the electron beam current is considered. The examined specimens are AlGaAs/GaAs laser heterostructures with InGaAs quantum well. It is shown that for low electron beam currents, which are typically used, the uniform cathodoluminescence is observed, while for the increasing high electron beam current the oval defects become more and more visible. The influence of electrical properties of the structure on the luminescence detection is explained.
\end{abstract}

PACS numbers: 78.60.Hk, 85.30.De, 61.72.-y

\section{Introduction}

Cathodoluminescence (CL) technique performed in scanning electron microscopes (SEM) is widely applied to determine the local spectrum of luminescence, map the optical activity in the semiconductor materials and structures, and revealing defects [1]. The present investigations show that the use of the appropriate value of electron beam (e-beam) current may be crucial for revealing semiconductor defects by means of CL technique.

\section{Experimental}

The examined specimens were $\mathrm{AlGaAs} / \mathrm{GaAs}$ laser heterostructures with the $8 \mathrm{~nm}$ thick InGaAs quantum well $(\mathrm{QW})$, manufactured with molecular beam epitaxy (MBE). The semiconductor layers in the regions of one mesa (on the left) and outside the mesa (on the right) are shown in Fig. 1a (not in the scale) and the corresponding electrical diagram related to the e-beam excitement in the region outside the mesa is shown in Fig. 1b. The structures were withdrawn from the technological process of laser manufacturing before its final stages and therefore have a large area with several individual mesas. Except for the regions below the mesas, $p$-AlGaAs laser-cladding was thinned by the etching process. The mesas were coated with gold in order to provide further electric connections. Some of the investigated structures have got a gold layer deposited also on their facets. The MonoCL2 (Oxford Instruments) system in Philips XL30 SEM was used for the CL detection.

\section{Method}

Electron beam generates in semiconductors electronhole $(\mathrm{e}-\mathrm{h})$ pairs, which can diffuse randomly and recombine radiatively or nonradiatively. In the former case,

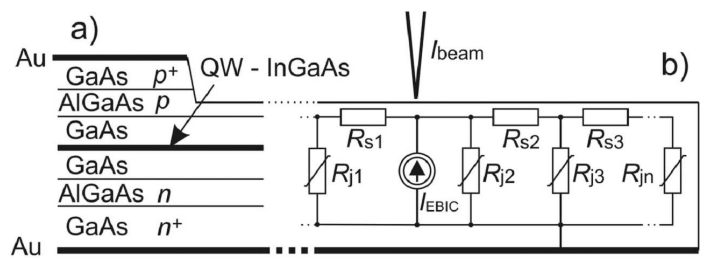

Fig. 1. (a) The semiconductor layers in the regions of the mesa (on the left) and outside the mesa (on the right), not in the scale. (b) The electrical diagram related to the e-beam excitement in the region outside the mesa.

photons are emitted, which can be detected by a CL detector in SEM. The presence of $p-n$ junction in the examined structures causes that the strong electric field separates the pairs that were generated within this region or diffused into it. If both sides of the junction are connected by an external electric circuit, then the electron beam induced current (EBIC, $I_{\mathrm{EBIC}}$ ) flows in this circuit and can be measured there. Even without the presence of an external circuit the rest of the structure constitutes an internal load for the $I_{\mathrm{EBIC}}$ current source (Fig. 1b). If local places with increased junction conductivity (e.g. shunts or defects) exist within the structure, then the generated $\mathrm{e}-\mathrm{h}$ pairs may outflow through lateral resistances to these places and do not produce light emission. This effect decreases the detected CL signal [2]. For example, CL measurements performed for structures of two types, with and without gold deposition on their facets (which leads to the junction short circuiting), show strong quenching of the CL signal generated in QW (i.e. about $990 \mathrm{~nm}$ wavelength) in the structures with gold.

When such sites of increased junction conductivity (of shunts or other defects) are absent, or the lateral resis- 
tance from e-beam to these sites is high, than the CL signal is unaffected. Therefore, the detected CL strongly depends on $I_{\text {beam }}$ value. Namely, the resistance of $p-n$ junction (which due to $e-h$ pair separation is forward biased) strongly depends on the junction current. With no external electrical circuit (i.e. in the case typical of CL studies) the junction current is constituted by EBIC, which increases with $I_{\text {beam. }}$. This increase of EBIC is linear for low $I_{\text {beam }}$ and it saturates for higher $I_{\text {beam }}$ values [3]. For adequately low $I_{\text {beam }}$ values the junction resistance is high comparatively to the lateral resistances, thus the current generated by e-beam may flow all over the structure. In this case, the presence of any shunts or defects in the structure affects the CL signal, therefore no CL contrast is detected between the defected areas and the rest of the structure. On the contrary, for high $I_{\text {beam }}$ values the junction resistance diminishes and becomes comparable to the lateral resistances between the e-beam and remote defects. In such a case the presence of distant shunts or defects does not affect the measured CL signal or affects it much less.

\section{Results and discussion}

Several monochromatic CL images of the examined structures were registered for the wavelength of $990 \mathrm{~nm}$ (the maximum of the QW emission peak) and different values of $I_{\text {beam }}$. Three of them were shown in Fig. 2. It is visible that for $I_{\text {beam }}$ of about $100 \mathrm{nA}$ (Fig. 2a) there is approximately uniform distribution of the CL signal over most of the structure. The dark region on the left side of the images represents the mesa coated with gold, which strongly decreases the e-beam penetration and limits the CL signal. With the increase of $I_{\text {beam }}$ (Figs. 2b, c) the defects present in the structure become more and more visible.

The lack of contrast of the defects in the case of the lowest $I_{\text {beam }}$ (Fig. 2a) is caused by the flow of internal EBIC all over the structure at such a low $I_{\text {beam }}$ (independently of the e-beam location), which quenches the CL signal. While for high $I_{\text {beam }}$ the CL signal is unaffected by the EBIC, i.e. the defects are well visible (Fig. 2c), for intermediate $I_{\text {beam }}$ values (Fig. $2 \mathrm{~b}$ ) some (e.g. stronger) defects are much more visible than the others (e.g. weaker ones), i.e. the visibility of defects depends on $I_{\text {beam }}$.

Another explanation for the defect detection changing with $I_{\text {beam }}$ can be considered, which can partially account for the observed effect. Namely, if the internal EBIC that flows from the e-beam position to remote defects is responsible for the emission of electroluminescence (EL), then this luminescence can be also measured by the CL detector.

The observations of our samples in SEM confirmed that the peaks of CL and EL spectra for the investigated structures occur at the same wavelengths. The EL signal at room temperature for forward currents in similar InGaAs/GaAs laser diodes can be easily detected for injection current densities that may be comparable
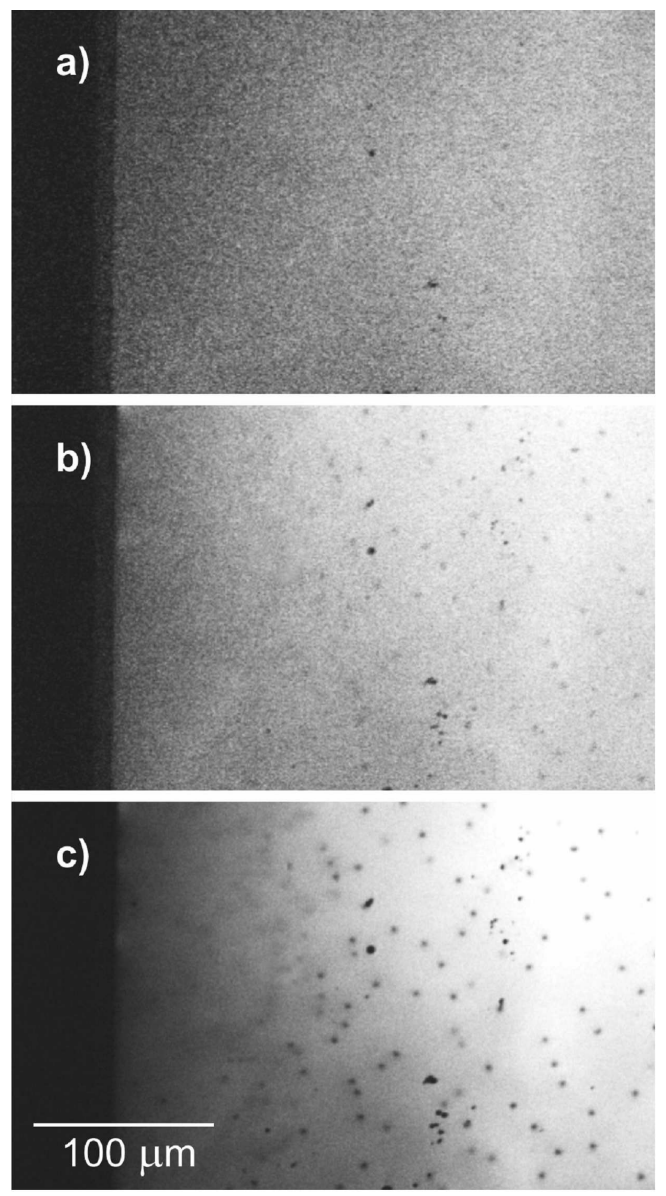

Fig. 2. CL monochromatic images of an exemplary structure at the wavelength corresponding to emission from QW (at $990 \mathrm{~nm}$ ) for $I_{\text {beam }}$ of (a) $100 \mathrm{nA}$, (b) $179 \mathrm{nA}$, (c) $285 \mathrm{nA}$.

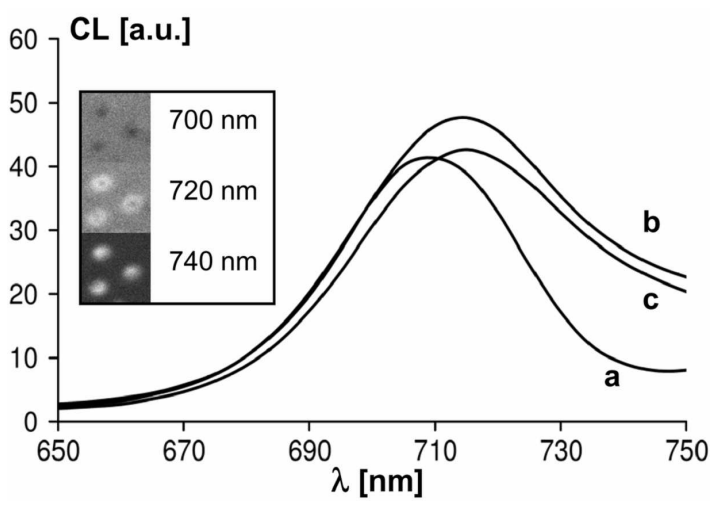

Fig. 3. The peak of CL spectrum corresponding to the emission of AlGaAs layer, registered (a) outside the defect, (b) near the defect, (c) inside the defect. The inset shows monochromatic CL images of three exemplary defects taken at three different wavelengths. 
with current densities in local junction sites of our CL-measured structures [4]. The similarity of EL and CL spectra in the case of GaAs laser diodes has been attributed to the case of a single QW [5]. The defects observed in the investigated structures were identified as oval defects. The luminance spectra at QW wavelength (i.e. $990 \mathrm{~nm}$ ) in the areas of oval defects and outside them show a lack of any wavelength shift of this part of the spectrum but significant decrease of the CL intensity. The wavelength redshift visible at the wavelength of about $710 \mathrm{~nm}$ (Fig. 3) confirms that these oval defects in AlGaAs layers are Ga-enriched [6]. In the case of EL emission (due to EBIC current flow through $p-n$ junction) in the sites of such defects distant from the e-beam position, the share of EL and CL in the detected signal depends on $I_{\text {beam }}$. It leads to the increasing visibility of the defects with the increasing $I_{\text {beam }}$.

\section{Conclusions}

What was described is the dependence between the defect detection with use of CL and the value of $I_{\text {beam }}$ applied in the SEM observation. As it was shown for $\mathrm{AlGaAs} / \mathrm{GaAs}$ structures with InGaAs QW, the influence of oval defects on the QW emission becomes visible at $I_{\text {beam }}$ values much higher than the ones typically used in CL investigations of similar laser diodes [7].
Although the CL measurements are often performed for specimens cooled to low temperatures in order to decrease irradiative recombination, when any shunts are present in the investigated structure, the cooling of a specimen cannot ensure that the CL signal remains unchanged, while the increase in $I_{\text {beam }}$ value may prevent the quenching of the CL-signal.

\section{References}

[1] A. Gustaffson, M.E. Pistol, L. Montelius, L. Samuelson, J. Appl. Phys. 84, 1715 (1998).

[2] A. Czerwinski, M. Pluska, J. Ratajczak, A. Szerling, J. Kątcki, Appl. Phys. Lett. 93, 041109 (2008).

[3] A. Czerwinski, M. Pluska, J. Ratajczak, A. Szerling, J. Kątcki, Appl. Phys. Lett. 89, 241919 (2006).

[4] G. Beister, J. Maege, G. Erbert G. Trankle, Solid-State Electron. 42, 1939 (1998).

[5] M. Krahl, N. Kirstaedter, R.K. Bauer, D. Bimberg, H.P. Meier, C. Harder, J. Appl. Phys. 70, 5561 (1991).

[6] A. Szerling, K. Kosiel, A. Wójcik-Jedlińska, M. Płuska, M. Bugajski, Mater. Sci. Semicond. Proc. 9, 25 (2006).

[7] O. Sjölund, H.T. Lin, D.H. Rich, M. Ghisoni, A. Larsson, S. Wang, J. Thordsson, T.G. Andersson, J. Appl. Phys. 82, 1438 (1997). 